\title{
The application of barium oesophagography in patients with suspected tracheo-oesophageal fistula: preferred or avoided?
}

\author{
To the Editor:
}

The review article by KIM et al. [1] presents excellent work in the evolving management of tracheo-oesophageal fistula (TOF) in adults.

The goals of TOF management are to protect pulmonary function and treat soiling of the respiratory tract, which result in pneumonitis and, ultimately, pulmonary sepsis.

However, in the diagnosis of TOF, KIM et al. [1] recommend barium preferentially when performing oesophagography. Is barium really suitable for patients with suspected TOF?

Aspiration of barium contrast is a rare complication of gastrointestinal studies. In patients with TOF, barium flow to the airway causes aspiration through the pathological connection between the trachea and oesophagus.

Barium is an inert material that can cause different symptoms, from asymptomatic mechanical obstruction to serious symptoms of respiratory distress [2]. A slight amount of barium aspiration may cause no symptomatic effects. While large amounts of barium in the lungs may interfere with gas exchange leading to a shunt effect and an altered ventilation/perfusion ratio $[2,3]$. Furthermore, barium could provoke a local reaction in lung parenchyma and lead to neutrophil infiltrates in the alveolar spaces, which makes it a potentially life-threatening event because of its direct harmful effects on lung tissue [4].

In a literature review of barium aspiration during upper gastrointestinal examination, $75 \%$ of patients presented with dyspnoea, hypoxaemia, acute respiratory distress syndrome or respiratory failure; $36.36 \%$ of patients died due to barium aspiration and $9.10 \%$ of patients died of complications within 1 year [5].

In patients with suspected TOF, we recommend using a non-ionic isotonic contrast agent to perform oesophagography. Non-ionic isotonic contrast agents are highly hydrophilic, resulting in minimal chemical toxicity. They are non-ionic and, therefore, eliminate coulomb interactions in solutions formulated with their respective humoral isotonic counterparts [6]. The application of non-ionic isotonic contrast agents in bronchography is safe and effective [7]. Although the cost of non-ionic isotonic contrast agents is greater than barium, it protects the lung function from barium destruction.

In brief, when performing oesophagography in patients with suspected TOF, non-ionic isotonic contrast agent should be encouraged and barium should be avoided.

Yahua Li, Huibin Lu, Kewei Ren, Xinwei Han and Jianzhuang Ren

Dept of Interventional Radiology, The First Affiliated Hospital of Zhengzhou University, Zhengzhou, China.

Correspondence: Xinwei Han, Dept of Interventional Radiology, The First Affiliated Hospital of Zhengzhou University, No.1 Jianshe Road, Zhengzhou 450052, China. E-mail: fcchanxw@zzu.edu.cn

Received: 7 Nov 2020 | Accepted: 14 Nov 2020

Provenance: Submitted article, peer reviewed.

@ERSpublications

When performing oesophagography in patients with suspected tracheo-oesophageal fistula, non-ionic isotonic contrast agent should be encouraged and barium avoided https://bit.ly/2IOl7p6

Cite this article as: $\mathrm{Li} \mathrm{Y,} \mathrm{Lu} \mathrm{H,} \mathrm{Ren} \mathrm{K}$, et al. The application of barium oesophagography in patients with suspected tracheo-oesophageal fistula: preferred or avoided? Eur Respir Rev 2021; 30: 200358 [https://doi. org/10.1183/16000617.0358-2020]. 
Conflict of interest: None declared.

\section{References}

1 Kim HS, Khemasuwan D, Diaz-Mendoza J, et al. Management of tracheo-oesophageal fistula in adults. Eur Respir Rev 2020; 29: 200094.

2 Fuentes Santos C, Steen B. Aspiration of barium contrast. Case Rep Pulmonol 2014; 2014: 215832.

3 Zhang L, Yang Y, Zhang J, et al. Barium sulfate aspiration: severe chemical pneumonia induced by a massive reflux of contrast medium during small bowel barium enema. Forensic Sci Int 2015; 253: e16-e19. Whiting J. Aspiration of barium. N Engl J Med 2003; 348: 2582-258.

5 Yan GW, Deng JF, Bhetuwal A, et al. A case report and literature review of barium sulphate aspiration during upper gastrointestinal examination. Medicine (Baltimore) 2017; 96: e8821.

6 Dawson P. The non-ionic isotonic contrast agents. Perspectives and controversies. Eur Radiol 1996; 6 Suppl 2: S20-S24.

7 Li Y, Zhu M, Chen J, et al. Single Y-shaped tracheal self-expandable metallic stent for emergent carinal stenosis combined with stenosis of the right main and intermediate bronchi. Medicine (Baltimore) 2020; 99: e20498. 